PROCEEDINGS OF THE

AMERICAN MATHEMATICAL SOCIETY

Volume 138, Number 1, January 2010, Pages 189-197

S 0002-9939(09)10073-4

Article electronically published on August 19, 2009

\title{
COMMUTING TOEPLITZ OPERATORS ON THE HARDY SPACE OF THE POLYDISK
}

\author{
YOUNG JOO LEE
}

(Communicated by Marius Junge)

\begin{abstract}
We study the commuting problem for Toeplitz operators on the Hardy space of the polydisk. We consider two symbols where one is an arbitrarily bounded symbol and the other is a bounded symbol which has the form of a sum of an analytic function and a co-analytic function. We then give a necessary and sufficient condition on the symbols for which the corresponding Toeplitz operators commute each other.
\end{abstract}

\section{INTRODUCTION}

Let $T$ be the boundary of the unit disk $D$ in the complex plane $\mathbb{C}$. The polydisk $D^{n}$ and torus $T^{n}$ are the cartesian products of $n$ copies of $D$ and $T$, respectively. We let $L^{p}\left(T^{n}\right)=L^{p}\left(T^{n}, \sigma\right)$ denote the usual Lebesgue space on $T^{n}$ where $\sigma=\sigma_{n}$ is the normalized Haar measure on $T^{n}$. The Hardy space $H^{2}\left(D^{n}\right)$ is the closure of the polynomials in $L^{2}\left(T^{n}\right)$. As is well known, we can identify functions in $H^{2}\left(D^{n}\right)$ with its holomorphic extension to $D^{n}$ via the Poisson extension. Thus, we will use the same notation for a function $f \in H^{2}\left(D^{n}\right)$ and its holomorphic extension $f$ on $D^{n}$. Let $P$ denote the orthogonal projection from $L^{2}\left(T^{n}\right)$ onto $H^{2}\left(D^{n}\right)$. For a function $u \in L^{\infty}\left(T^{n}\right)$, the Toeplitz operator $T_{u}$ with symbol $u$ is defined by

$$
T_{u} f=P(u f)
$$

for functions $f \in H^{2}\left(D^{n}\right)$. Then, clearly, $T_{u}$ is a bounded linear operator on $H^{2}\left(D^{n}\right)$.

In this paper, we study the commuting problem of when two Toeplitz operators commute each other. On the Hardy space of the unit disk, Brown and Halmos [2] first obtained a complete description of bounded symbols of commuting Toeplitz operators by asserting that two Toeplitz operators with bounded symbols commute on $H^{2}(D)$ if and only if both symbols are all analytic or both symbols are all coanalytic, or a nontrivial linear combination of the symbols is constant. Here and in what follows, a function in $L^{1}\left(T^{n}\right)$ is said to be analytic if all its Fourier coefficients vanish outside $\mathbb{Z}_{+}^{n}$, where $\mathbb{Z}_{+}$is the set of all nonnegative integers. Also, the complex conjugate of an analytic function is called co-analytic. In [10, Stroethoff gave a new proof to prove the result of Brown and Halmos as well as a generalization.

Received by the editors February 24, 2009.

2000 Mathematics Subject Classification. Primary 47B35; Secondary 32A35.

Key words and phrases. Toeplitz operators, Hardy space, polydisk.

(C)2009 American Mathematical Society Reverts to public domain 28 years from publication 
Also, Zheng [11] extended the result of Brown and Halmos to the Hardy space of the ball with bounded pluriharmonic symbols.

Also, the same commuting problem has been studied on the polydisk. In a recent paper, the author 8 studied the same problem on the bidisk when one symbol is a general bounded symbol and the other is a certain type of bounded symbol. On the general polydisk, Choe, Koo and the author 3] characterized two pluriharmonic symbols of commuting Toeplitz operators in the course of studying the corresponding problem on the Bergman space. The result in [3] shows that for given functions $f, g, h, k \in H^{2}\left(D^{n}\right), T_{f+\bar{g}}$ and $T_{h+\bar{k}}$ commute on $H^{2}\left(D^{n}\right)$ if and only if $f \bar{k}-h \bar{g}$ is $n$-harmonic on $D^{n}$. Recall that a function $u \in C^{2}\left(D^{n}\right)$ is called $n$-harmonic, as in 9], if $u$ is harmonic in each variable separately. More explicitly, $u$ is $n$-harmonic if

$$
\partial_{j} \bar{\partial}_{j} u=0, \quad j=1,2, \ldots, n,
$$

where $\partial_{j}=\partial / \partial z_{j}$.

In this paper, we continue to study the same commuting problem on the polydisk for Toeplitz operators with two symbols where one is an arbitrarily bounded symbol and the other is a bounded symbol which has the form of $f+\bar{g}$ where $f$ and $g$ are analytic on $T^{n}$. We then give a characterization for which the corresponding Toeplitz operators commute.

The following is the our main result.

Main Theorem. Let $u, v \in L^{\infty}\left(T^{n}\right)$. Suppose $v=f+\bar{g}$ where $f, g$ are analytic on $T^{n}$. Then the following statements are equivalent:

(a) $T_{u} T_{v}=T_{v} T_{u}$ on $H^{2}\left(D^{n}\right)$.

(b) $\widehat{u}[f-\bar{g}]$ is n-harmonic on $D^{n}$.

Here $\widehat{u}$ is the Poisson integral of $u$.

We were not able to obtain a characterization when two symbols are all arbitrarily bounded symbols. Our result generalizes the results in [2] and [3] mentioned above. To prove the Main Theorem, we use an entirely different argument from those of [2] and [3]. In the next section, we collect some basic facts. In Section 3, we prove the Main Theorem and recover the known results as immediate consequences.

\section{Preliminaries}

Recall that we can identify functions in $H^{2}\left(D^{n}\right)$ with its holomorphic extension on $D^{n}$ via the Poisson extension. With this identification, the point evaluation at each point in $D^{n}$ is a bounded linear functional on $H^{2}\left(D^{n}\right)$. Hence, for each $a \in D^{n}$, there exists a unique kernel function $K_{a} \in H^{2}\left(D^{n}\right)$ which has reproducing property

$$
f(a)=\left\langle f, K_{a}\right\rangle
$$

for functions $f \in H^{2}\left(D^{n}\right)$. Here and in what follows, the notation $\langle$,$\rangle denotes the$ inner product in $L^{2}\left(T^{n}\right)$ with respect to the measure $\sigma$. For $a=\left(a_{1}, \cdots, a_{n}\right) \in D^{n}$, it is well known that $K_{a}$ is the Cauchy kernel whose the explicit formula is given by

$$
K_{a}(\zeta)=\prod_{j=1}^{n} \frac{1}{\left(1-\overline{a_{j}} \zeta_{j}\right)}, \quad \zeta=\left(\zeta_{1}, \cdots, \zeta_{n}\right) \in T^{n}
$$


Thus, by (1), we can write the projection $P$ as the integral operator

$$
P \varphi(a)=\left\langle P \varphi, K_{a}\right\rangle=\left\langle\varphi, K_{a}\right\rangle=\int_{T^{n}} \frac{\varphi(\zeta)}{\prod_{j=1}^{n}\left(1-a_{j} \overline{\zeta_{j}}\right)} d \sigma(\zeta)
$$

for $\varphi \in L^{2}\left(T^{n}\right)$ and $a=\left(a_{1}, \cdots, a_{n}\right) \in T^{n}$. The above integral formula for $P$ shows that the domain of $P$ can be extended to $L^{1}\left(T^{n}\right)$. Moreover, it is known that $P f=f$ for analytic functions $f \in L^{1}\left(T^{n}\right)$. Also, since $P$ is the projection from $L^{2}\left(T^{n}\right)$ onto $H^{2}\left(D^{n}\right)$, we have

$$
P[\bar{f} P(\varphi)](a)=\int_{T^{n}} P(\varphi) \overline{f K_{a}} d \sigma=\int_{T^{n}} \varphi \overline{f K_{a}} d \sigma=P(\bar{f} \varphi)(a)
$$

for points $a \in D^{n}$ and hence

$$
P[\bar{f} P(\varphi)]=P(\bar{f} \varphi)
$$

for functions $f \in H^{2}\left(D^{n}\right)$ and $\varphi \in L^{\infty}\left(T^{n}\right)$. Also, note that

$$
P\left(\bar{f} K_{a}\right)(z)=\left\langle K_{a}, f K_{z}\right\rangle=\overline{f(a) K_{z}(a)}=\overline{f(a)} K_{a}(z), \quad z \in D^{n},
$$

and then we have

$$
P\left(\bar{f} K_{a}\right)=\overline{f(a)} K_{a}
$$

for functions $f \in H^{2}\left(D^{n}\right)$ and points $a \in D^{n}$. In particular, for $n=1$, we have

$$
\int_{T} \frac{\bar{\phi}(\xi)}{(1-\lambda \bar{\xi})(1-\bar{\mu} \xi)} d \sigma_{1}(\xi)=\frac{\overline{\phi(\mu)}}{(1-\bar{\mu} \lambda)}
$$

for $\phi \in H^{2}(D)$ and points $\lambda, \mu \in D$.

For $a=\left(a_{1}, \cdots, a_{n}\right) \in D^{n}$, we let $k_{a}$ be the normalized kernel given by

$$
k_{a}(\zeta)=\prod_{j=1}^{n} \frac{\sqrt{1-\left|a_{j}\right|^{2}}}{\left(1-\overline{a_{j}} \zeta_{j}\right)}
$$

for $\zeta=\left(\zeta_{1}, \cdots, \zeta_{n}\right) \in T^{n}$. Since $\left|k_{a}\right|^{2}$ is the Poisson kernel for $D^{n}$, the Poisson integral $\widehat{\varphi}$ of $\varphi \in L^{1}\left(T^{n}\right)$ can be written as

$$
\widehat{\varphi}(a)=\int_{T^{n}} \varphi\left|k_{a}\right|^{2} d \sigma, \quad a \in D^{n} .
$$

As is well known, each Poisson integral is $n$-harmonic on $D^{n}$. Given a function $\psi$ on $D^{n}$, the radial limit $\psi^{*}$ is defined by $\psi^{*}(\zeta)=\lim _{r \rightarrow 1} \psi(r \zeta)$ at every $\zeta \in T^{n}$ at which the limit exists. For $\varphi \in L^{1}\left(T^{n}\right)$, it is known that $[\widehat{\varphi}]^{*}=\varphi$ a.e on $T^{n}$, and we note that

$$
\widehat{\varphi}(a)=\left\langle\varphi k_{a}, k_{a}\right\rangle=\frac{1}{K_{a}(a)} P\left(\varphi K_{a}\right)(a)
$$

for points $a \in D^{n}$. See Chapter 2 of $[9$ for related facts and details.

\section{The proof of the Main Theorem}

In this section, we prove the Main Theorem and then derive the known results as immediate consequences. Before doing this, we need a couple of preliminary lemmas. We first need the following characterization of harmonicity of functions which is a finite sum of products of holomorphic and antiholomorphic functions on the unit disk $D$. 
Lemma 1. Let $f_{1}, \cdots, f_{N}$ and $k_{1}, \cdots, k_{N}$ be holomorphic functions on $D$ for which $\sum_{j=1}^{N} f_{j} \overline{k_{j}}$ is harmonic on $D$. Then we have

$$
\sum_{j=1}^{N} f_{j} \overline{k_{j}}=\sum_{j=1}^{N}\left[f_{j} \overline{k_{j}(0)}+f_{j}(0) \overline{k_{j}}-f_{j}(0) \overline{k_{j}(0)}\right] .
$$

Proof. See Lemma 2 of 8 .

We also need the following lemma which is taken from Lemma 9 of [4].

Lemma 2. Let $f_{1}, \cdots, f_{N}$ and $k_{1}, \cdots, k_{N}$ be finitely many holomorphic functions on D. If $\sum_{j=1}^{N} f_{j} \overline{k_{j}}=0$ on $D$, then

$$
\sum_{j=1}^{N} f_{j}(\lambda) \overline{k_{j}(\mu)}=0
$$

for every $\lambda, \mu \in D$.

We are now ready to prove the Main Theorem.

Proof of the Main Theorem. Since $v \in L^{\infty}\left(T^{n}\right)$, we note that $f, g \in H^{2}\left(D^{n}\right)$. Suppose (a) and let $a \in D^{n}$. Since $T_{u} T_{v}=T_{v} T_{u}$, we have in particular,

$$
\left\langle T_{v} T_{u} k_{a}, k_{a}\right\rangle=\left\langle T_{u} T_{v} k_{a}, k_{a}\right\rangle .
$$

By (2), we first note that $P\left[\bar{g} P\left(u k_{a}\right)\right]=P\left(\bar{g} u k_{a}\right)$ and hence

$$
T_{v} T_{u} k_{a}=P\left[(f+\bar{g}) P\left(u k_{a}\right)\right]=f P\left(u k_{a}\right)+P\left(\bar{g} u k_{a}\right) .
$$

Since $f \in H^{2}\left(D^{n}\right)$, we note by (5)

$$
\left\langle f P\left(u k_{a}\right), k_{a}\right\rangle=\frac{1}{K_{a}(a)} f(a) P\left(u K_{a}\right)(a)=f(a) \widehat{u}(a)
$$

and $\left\langle P\left[\bar{g} u k_{a}\right], k_{a}\right\rangle=\widehat{[\bar{g} u]}(a)$. It follows that

$$
\begin{aligned}
\left\langle T_{v} T_{u} k_{a}, k_{a}\right\rangle & =\left\langle f P\left(u k_{a}\right), k_{a}\right\rangle+\left\langle P\left(\bar{g} u k_{a}\right), k_{a}\right\rangle \\
& =f(a) \widehat{u}(a)+\widehat{[\bar{g} u]}(a) .
\end{aligned}
$$

On the other hand, by (3), we have that $T_{f+\bar{g}} k_{a}=[f+\overline{g(a)}] k_{a}$ and hence

$$
T_{u} T_{v} k_{a}=T_{u} T_{f+\bar{g}} k_{a}=P\left(u f k_{a}\right)+\overline{g(a)} P\left(u k_{a}\right) .
$$

It follows from (5) that

$$
\left\langle T_{u} T_{v} k_{a}, k_{a}\right\rangle=\widehat{[u f]}(a)+\overline{g(a)} \widehat{u}(a) .
$$

Combining the above with (8) and together with (6), we have

$$
\widehat{u}[f-\bar{g}]=[\widehat{u f-\bar{g}} u]
$$

on $D^{n}$. Since $[\widehat{u f-\bar{g}} u]$ is $n$-harmonic, we see that the function $\widehat{u}[f-\bar{g}]$ is also $n$-harmonic on $D^{n}$. Thus we have (b).

Now suppose (b) and prove (a). In the proof, we will use the usual multi-index notations. The notation $\mathbb{Z}$ denotes the set of all integers. For $\zeta=\left(\zeta_{1}, \cdots, \zeta_{n}\right) \in T^{n}$ and $\alpha=\left(\alpha_{1}, \cdots, \alpha_{n}\right) \in \mathbb{Z}^{n}$, we write $\zeta^{\alpha}=\zeta_{1}^{\alpha_{1}} \zeta_{2}^{\alpha_{2}} \cdots \zeta_{n}^{\alpha_{n}}$ as usual. Also, let $J=\{0,1\}$. 
Let us consider the multiple Fourier series for $u$ given by

$$
u(\zeta)=\sum_{\alpha \in \mathbb{Z}^{n}} \hat{u}(\alpha) \zeta^{\alpha}, \quad \zeta=\left(\zeta_{1}, \cdots, \zeta_{n}\right) \in T^{n},
$$

where $\hat{u}(\alpha)$ are Fourier coefficients of $u$. Given $I=\left(i_{1}, \cdots, i_{n}\right)$, where each $i_{k} \in J$, we let $[I]$ be the set of all $\alpha=\left(\alpha_{1}, \cdots, \alpha_{n}\right) \in \mathbb{Z}^{n}$ for which $\alpha_{k} \leq 0$ if $i_{k}=0$, and $\alpha_{k}>0$ if $i_{k}=1$ for each $k$. Put

$$
u_{I}(\zeta)=\sum_{\alpha \in[I]} \hat{u}(\alpha) \zeta^{\alpha}
$$

Note that for each $k=1, \cdots, n$, if $i_{k}=1$ (resp. $\left.i_{k}=0\right)$, then the function $u_{I}(\zeta)$ is analytic (resp. co-analytic) in the variable $\zeta_{k}$. For each $\alpha=\left(\alpha_{1}, \cdots, \alpha_{n}\right) \in[I]$ and $z=\left(z_{1}, \cdots, z_{n}\right) \in D^{n}$, we define

$$
\tilde{z}_{k}^{\alpha_{k}}=\left\{\begin{array}{ll}
\overline{z_{k}^{-\alpha_{k}}} & \text { if } i_{k}=0, \\
z_{k}^{\alpha_{k}} & \text { if } i_{k}=1,
\end{array} \quad k=1, \cdots, n,\right.
$$

and let $\tilde{z}^{\alpha}=\tilde{z}_{1}^{\alpha_{1}} \cdots \tilde{z}_{n}^{\alpha_{n}}$. Define a function $\mathbf{U}_{I}$ on $D^{n}$ by

$$
\mathbf{U}_{I}(z)=\sum_{\alpha \in[I]} \widehat{u}(\alpha) \tilde{z}^{\alpha}, \quad z=\left(z_{1}, \cdots, z_{n}\right) \in D^{n} .
$$

Then, for each $k$, if $i_{k}=1$ (resp. $i_{k}=0$ ), the function $\mathbf{U}_{I}(z)$ is holomorphic (resp. antiholomorphic) in the variable $z_{k}$. Also, by (4) and an application of Corollary 2.3 of [6], we can easily verify

$$
P\left(u_{I} K_{a}\right)(z)=\mathbf{U}_{I}\left(\left(1-i_{1}\right) a_{1}+i_{1} z_{1}, \cdots,\left(1-i_{n}\right) a_{n}+i_{n} z_{n}\right) K_{a}(z)
$$

for $a=\left(a_{1}, \cdots, a_{n}\right) \in D^{n}$. Thus, by (5) we have $\widehat{u_{I}}=\mathbf{U}_{I}$. Since the multiple Fourier series for $u$ can be rewritten as

$$
u=\sum_{i_{1}, \cdots, i_{n} \in J} u_{i_{1}, \cdots, i_{n}}
$$

we have

$$
\widehat{u}=\sum_{i_{1}, \cdots, i_{n} \in J} \mathbf{U}_{i_{1}, \cdots, i_{n}} .
$$

Put $\Psi=\widehat{u}[f-\bar{g}]$. Since $\Psi$ is $n$-harmonic on $D^{n}$ by the assumption, we see that

$$
\Psi=f \sum_{i_{1}, \cdots, i_{n} \in J} \mathbf{U}_{i_{1}, \cdots, i_{n}}-\bar{g} \sum_{i_{1}, \cdots, i_{n} \in J} \mathbf{U}_{i_{1}, \cdots, i_{n}}
$$

is $n$-harmonic on $D^{n}$. For each $k=1, \cdots, n$, we recall that if $i_{k}=1$ (resp. $i_{k}=0$ ), then the function $\mathbf{U}_{i_{1}, \cdots, i_{n}}(z)$ is holomorphic (resp. antiholomorphic) in the variable $z_{k}$. Thus, the function $\Psi$ is a finite sum of products of a holomorphic function and an antiholomorphic function in each variable separately. Also, since $\Psi$ is $n$-harmonic on $D^{n}, \Psi$ is harmonic in each variable separately. Given $i, j \in J$, we put

$$
\vartheta(i, j)=\left\{\begin{aligned}
1 & \text { if }(i, j)=(1,0),(0,1) \text { or }(1,1) \\
-1 & \text { if }(i, j)=(0,0)
\end{aligned}\right.
$$


Now, applying Lemma 1 to $\Psi$ with respect to the first variable, we see that

$$
\begin{aligned}
\Psi(a)= & f(a) \sum_{i_{2}, \cdots, i_{n} \in J} \mathbf{U}_{1, i_{2}, \cdots, i_{n}}(a)-\overline{g(a)} \sum_{i_{2}, \cdots, i_{n} \in J} \mathbf{U}_{0, i_{2}, \cdots, i_{n}}(a) \\
& +f(a) \sum_{i_{2}, \cdots, i_{n} \in J} \mathbf{U}_{0, i_{2}, \cdots, i_{n}}(a)-\overline{g(a)} \sum_{i_{2}, \cdots, i_{n} \in J} \mathbf{U}_{1, i_{2}, \cdots, i_{n}}(a) \\
= & f(a) \sum_{i_{2}, \cdots, i_{n} \in J} \mathbf{U}_{1, i_{2}, \cdots, i_{n}}(a)-\overline{g(a)} \sum_{i_{2}, \cdots, i_{n} \in J} \mathbf{U}_{0, i_{2}, \cdots, i_{n}}(a) \\
& +\sum_{\substack{l_{1}, m_{1} \in J \\
l_{1} m_{1}=0}} \vartheta\left(l_{1}, m_{1}\right) f\left(l_{1} a_{1}, a^{\prime}\right) \sum_{i_{2}, \cdots, i_{n} \in J} \mathbf{U}_{0, i_{2}, \cdots, i_{n}}\left(m_{1} a_{1}, a^{\prime}\right) \\
& -\sum_{l_{1}, m_{1} \in J} \vartheta\left(l_{1}, m_{1}\right) \overline{g\left(m_{1} a_{1}, a^{\prime}\right)} \sum_{i_{2}, \cdots, i_{n} \in J} \mathbf{U}_{1, i_{2}, \cdots, i_{n}}\left(l_{1} a_{1}, a^{\prime}\right) \\
= & \sum_{l_{1}, m_{1} \in J} \vartheta\left(l_{1}, m_{1}\right) f\left(l_{1} a_{1}, a^{\prime}\right) \sum_{i_{2}, \cdots, i_{n} \in J} \mathbf{U}_{l_{1} m_{1}, i_{2}, \cdots, i_{n}}\left(m_{1} a_{1}, a^{\prime}\right) \\
& -\sum_{l_{1}, m_{1} \in J} \vartheta\left(l_{1}, m_{1}\right) \overline{g\left(m_{1} a_{1}, a^{\prime}\right)} \sum_{i_{2}, \cdots, i_{n} \in J} \mathbf{U}_{\left(1-l_{1} m_{1}\right), i_{2}, \cdots, i_{n}}\left(l_{1} a_{1}, a^{\prime}\right),
\end{aligned}
$$

where $a^{\prime}=\left(a_{2}, \cdots, a_{n}\right)$ and $a=\left(a_{1}, a^{\prime}\right) \in D^{n}$. Repeating the same argument as above with respect to variables $a_{2}, \cdots, a_{n}$ in order, we see that the $n$-harmonicity of $\Psi$ implies that $\Psi$ admits the following decomposition:

$$
\Psi(a)=\sum_{l_{k}, m_{k} \in J} \vartheta_{l, m}\left[f(l \cdot a) \mathbf{U}_{l_{1} m_{1}, \cdots, l_{n} m_{n}}(m \cdot a)-\overline{g(m \cdot a)} \mathbf{U}_{1-l_{1} m_{1}, \cdots, 1-l_{n} m_{n}}(l \cdot a)\right],
$$

where we use the notation $l \cdot a=\left(l_{1} a_{1}, \cdots, l_{n} a_{n}\right), m \cdot a=\left(m_{1} a_{1}, \cdots, m_{n} a_{n}\right)$ and $\vartheta_{l, m}=\vartheta\left(l_{1}, m_{1}\right) \cdots \vartheta\left(l_{n}, m_{n}\right)$ for simplicity. Recall that

$$
\Psi=[f-\bar{g}] \sum_{i_{1}, \cdots, i_{n} \in J} \mathbf{U}_{i_{1}, \cdots, i_{n}} .
$$

Note that $\Psi$ is a finite sum of products of a holomorphic function and an antiholomorphic function in each variable separately. Also, we see that the function on the right-hand side of (12) is also a finite sum of products of a holomorphic function and an antiholomorphic function in each variable separately. Hence, by (12) and an application of Lemma 2, we have

$$
\begin{aligned}
& {[f(z)-\overline{g(a)}] \sum_{i_{k} \in J} \mathbf{U}_{i_{1}, \cdots, i_{n}}\left(\left(1-i_{1}\right) a_{1}+i_{1} z_{1}, \cdots,\left(1-i_{n}\right) a_{n}+i_{n} z_{n}\right)} \\
& \quad=\sum_{l_{k}, m_{k} \in J} \vartheta_{l, m}\left[f(l z) \mathbf{U}_{l_{1} m_{1}, \cdots, l_{n} m_{n}}(\mathbf{P})-\overline{g(m a)} \mathbf{U}_{1-l_{1} m_{1}, \cdots, 1-l_{n} m_{n}}(\mathbf{Q})\right]
\end{aligned}
$$

for all $a, z \in D^{n}$. Here we use the notation

$$
\begin{aligned}
& \mathbf{P}=\left(\left[\left(1-l_{1} m_{1}\right) a_{1}+\left(l_{1} m_{1}\right) z_{1}\right] m_{1}, \cdots,\left[\left(1-l_{n} m_{n}\right) a_{n}+\left(l_{1} m_{1}\right) z_{n}\right] m_{n}\right), \\
& \mathbf{Q}=\left(\left[\left(1-l_{1} m_{1}\right) z_{1}+\left(l_{1} m_{1}\right) a_{1}\right] l_{1}, \cdots,\left[\left(1-l_{n} m_{n}\right) z_{n}+\left(l_{1} m_{1}\right) a_{n}\right] l_{n}\right)
\end{aligned}
$$

for simplicity. 
Note that the set $\left\{K_{a}: a \in D^{n}\right\}$ spans a dense subset of $H^{2}\left(D^{n}\right)$. So, in order to prove (a), it is sufficient to show that $\left[T_{u} T_{v}-T_{v} T_{u}\right] K_{a}=0$ for all $a \in D^{n}$. Using (77) and (9), we see that $T_{u} T_{v}=T_{v} T_{u}$ is equivalent to

$$
[f(z)-\overline{g(a)}] P\left(u K_{a}\right)(z)=P\left[(f-\bar{g}) u K_{a}\right](z)
$$

for all $a, z \in D^{n}$. Let $a=\left(a, \cdots, a_{n}\right), z=\left(z_{1}, \cdots, z_{n}\right) \in D^{n}$ be arbitrary points. By (11) and (10), we first have

$$
P\left(u K_{a}\right)(z)=K_{a}(z) \sum_{i_{1}, \cdots, i_{n} \in J} \mathbf{U}_{i_{1}, \cdots, i_{n}}(\mathbf{R})
$$

and then

$$
[f(z)-\overline{g(a)}] P\left(u K_{a}\right)(z)=[f(z)-\overline{g(a)}] K_{a}(z) \sum_{i_{1}, \cdots, i_{n} \in J} \mathbf{U}_{i_{1}, \cdots, i_{n}}(\mathbf{R})
$$

for all $a, z \in D^{n}$. Here, $\mathbf{R}=\left(\left(1-i_{1}\right) a_{1}+i_{1} z_{1}, \cdots,\left(1-i_{n}\right) a_{n}+i_{n} z_{n}\right)$ for simplicity.

On the other hand, by applications of (4) and Corollary 2.3 of [6] again, we can verify that

$$
P\left[f(l \cdot) u_{l_{1} m_{1}, \cdots, l_{n} m_{n}}(m \cdot) K_{a}\right](z)=f(l \cdot z) \mathbf{U}_{l_{1} m_{1}, \cdots, l_{n} m_{n}}(\mathbf{P}) K_{a}(z)
$$

and

$$
P\left[\bar{g}(m \cdot) u_{1-l_{1} m_{1}, \cdots, 1-l_{n} m_{n}}(l \cdot) K_{a}\right](z)=\bar{g}(m \cdot a) \mathbf{U}_{1-l_{1} m_{1}, \cdots, 1-l_{n} m_{n}}(\mathbf{Q}) K_{a}(z)
$$

for each $l=\left(l_{1}, \cdots, l_{n}\right)$ and $m=\left(m_{1}, \cdots, m_{n}\right)$, where $l_{k}, m_{k} \in J$. Here $l \cdot z=$ $\left(l_{1} z_{1}, \cdots, l_{n} z_{n}\right)$ and $m \cdot a=\left(m_{1} a_{1}, \cdots, m_{n} a_{n}\right)$. Also, given functions $\psi$ on $T^{n}$ and $i=\left(i_{1}, \cdots, i_{n}\right)$ with each $i_{k} \in J$, we use the notation $\psi(i \cdot)$ to denote the function on $T^{n}$ defined by $\psi(i \cdot)(\zeta)=\psi\left(i_{1} \zeta_{1}, \cdots, i_{n} \zeta_{n}\right)$ for $\zeta=\left(\zeta_{1}, \cdots, \zeta_{n}\right) \in T^{n}$.

Now, since $\Psi=[f-\bar{g}] \widehat{u}$, the radial limit $\Psi^{*}$ of $\Psi$ is equal to $[f-\bar{g}] u$. Also, since $\mathbf{U}_{i_{1}, \cdots, i_{n}}=\widehat{u_{i_{1}, \cdots, i_{n}}}$, we have that $\mathbf{U}_{i_{1}, \cdots, i_{n}}^{*}=u_{i_{1}, \cdots, i_{n}}$ for each $i_{1}, \cdots, i_{n} \in J$. It follows from (12) and observations above that

$$
\begin{aligned}
P & {\left[(f-\bar{g}) u K_{a}\right](z) } \\
& =P\left[\Psi^{*} K_{a}\right](z) \\
& =\sum_{l_{k}, m_{k} \in J} \vartheta_{l, m} P\left(\left[f(l \cdot) u_{l_{1} m_{1}, \cdots, l_{n} m_{n}}(m \cdot)-\bar{g}(m \cdot) u_{1-l_{1} m_{1}, \cdots, 1-l_{n} m_{n}}(l \cdot)\right] K_{a}\right)(z) \\
& =\sum_{l_{k}, m_{k} \in J} \vartheta_{l, m}\left[f(l z) \mathbf{U}_{l_{1} m_{1}, \cdots, l_{n} m_{n}}(\mathbf{P})-\bar{g}(m a) \mathbf{U}_{1-l_{1} m_{1}, \cdots, 1-l_{n} m_{n}}(\mathbf{Q})\right] K_{a}(z) .
\end{aligned}
$$

Now, combining the above with (15) and together with (13), we have (14). The proof is complete.

We now close the paper with some immediate consequences of the Main Theorem. First, if one of the symbols of commuting Toeplitz operators is analytic, then we have the following result, which is a special case of Theorem 3.2 of 5 or Corollary 1 of [7.

Corollary 3. Let $u, v \in L^{\infty}\left(T^{n}\right)$. Suppose $v$ is analytic. Then $T_{u} T_{v}=T_{v} T_{u}$ on $H^{2}\left(D^{n}\right)$ if and only if for each $k=1, \cdots, n, v(\zeta)$ is constant or $u(\zeta)$ is analytic in variable $\zeta_{k}$. 
Proof. By the Main Theorem, we have $T_{u} T_{v}=T_{v} T_{u}$ if and only if $v \widehat{u}$ is $n$-harmonic on $D^{n}$. Using the same notation as in the proof of the Main Theorem, we have

$$
\widehat{u}=\sum_{i_{1}, \cdots, i_{n} \in J} \mathbf{U}_{i_{1}, \cdots, i_{n}} .
$$

Note that the $n$-harmonicity of $v \widehat{u}$ is equivalent to that for each $k=1, \cdots, n$,

$$
\partial_{k} v(z) \sum_{i_{m} \neq i_{k}} \bar{\partial}_{k} \mathbf{U}_{i_{1}, \cdots, i_{k-1}, 0, i_{k+1} \cdots, i_{n}}(z)=0, \quad z \in D^{n}
$$

which is equivalent to either $v$ being constant or each $\mathbf{U}_{i_{1}, \cdots, i_{k-1} 0, i_{k+1} \cdots, i_{n}}$ being constant in variable $z_{k}$. Thus, $n$-harmonicity of $v \widehat{u}$ is equivalent to that for each $k$, $v(z)$ is constant or $\widehat{u}(z)$ is holomorphic in variable $z_{k}$, which in turn is equivalent to $v(\zeta)$ being constant or $u(\zeta)$ being analytic in variable $\zeta_{k}$. The proof is complete.

As another simple application, we recover the results in [2] and [3] mentioned before.

Corollary 4. Let $u, v \in L^{\infty}\left(T^{n}\right)$. Suppose $v=f+\bar{g}$ and $u=h+\bar{k}$, where $f, g, h, k$ are analytic functions on $T^{n}$. Then $T_{u} T_{v}=T_{v} T_{u}$ on $H^{2}\left(D^{n}\right)$ if and only if $f \bar{k}-h \bar{g}$ is n-harmonic on $D^{n}$.

Proof. Note that $\widehat{u}=h+\bar{k}$. Hence, by the Main Theorem, we have that $T_{u}$ and $T_{v}$ commute if and only if $[f-\bar{g}][h+\bar{k}]$ is $n$-harmonic, which is equivalent to the $n$-harmonicity of $f \bar{k}-h \bar{g}$. The proof is complete.

Given functions $f, g, h, k$ holomorphic on the unit disk $D$, we let $v=f+\bar{g}$ and $u=h+\bar{k}$. Then one can verify that $f \bar{k}-h \bar{g}$ is harmonic on $D$ if and only if $u, v$ are all holomorphic, $u, v$ are all antiholomorphic, or a nontrivial linear combination of $u, v$ is constant. For example, see the proof of Theorem 1 of [1. Also, every $u \in L^{2}(T)$ can be decomposed as $u=f+\bar{g}$ for some $f, g \in H^{2}(D)$. Combining these facts with Corollary 4, we can recover the result of Brown and Halmos in [2] on the unit disk.

Corollary 5. Let $u, v \in L^{\infty}(T)$. Then $T_{u} T_{v}=T_{v} T_{u}$ on $H^{2}(D)$ if and only if $u$ and $v$ are all analytic, $u$ and $v$ are all co-analytic, or a nontrivial linear combination of $u$ and $v$ is constant on $T$.

\section{REFERENCES}

[1] S. Axler and Z̆. C̆učković, Commuting Toeplitz operators with harmonic symbols, Integr. Equ. Oper. Theory 14(1991), 1-11. MR.1079815 (92f:47018)

[2] A. Brown and P. Halmos, Algebraic properties of Toeplitz operators, J. Reine Angew. Math. 213(1964), 89-102. MR0160136(28:3350)

[3] B. R. Choe, H. Koo and Y. J. Lee, Commuting Toeplitz operators on the polydisk, Trans. Amer. Math. Soc. 356(2004), 1727-1749. MR2031039 (2004k:47054)

[4] B. R. Choe and Y. J. Lee, Pluriharmonic symbols of commuting Toeplitz operators, Illinois J. Math. 37(1993), 424-436. MR.1219648 (94i:47041)

[5] X. Ding, Products of Toeplitz operators on the polydisk, Integr. Equ. Oper. Theory 45(2003), 389-403. MR1971745 (2004b:47051)

[6] X. Ding, The finite sum of finite products of Toeplitz operators on the polydisk, J. Math. Anal. Appl. 320(2006), 464-481. MR2230483 (2007b:47072)

[7] C. Gu, Some algebraic properties of Toeplitz and Hankel operators on polydisk, Arch. Math. 80(2003), 393-405. MR1982839 (2004e:47039)

[8] Y. J. Lee, Commuting Toeplitz operators on the Hardy space of the bidisk, J. Math. Anal. Appl. 341(2008), 738-749. MR.2394121 (2009c:47037) 
[9] W. Rudin, Function theory in polydiscs, W. A. Benjamin, Inc., 1969. MR0255841 (41:501)

[10] K. Stroethoff, Algebraic properties of Toeplitz operators on the Hardy space via the Berezin transform, Contemp. Math., 232, Amer. Math. Soc., Providence, RI, 1999, 313-319. MR1678343 (2000a:47060)

[11] D. Zheng, Commuting Toeplitz operators with pluriharmonic symbols, Trans. Amer. Math. Soc. 350(1998), 1595-1618. MR.1443898 (98i:47027)

Department of Mathematics, Chonnam National University, Gwangju 500-757, REPUBLIC OF KoREA

E-mail address: leeyj@chonnam.ac.kr 